\title{
In Vitro Test of Phagocytic Ability of Human Platelets Using Colloidal Carbon
}

\author{
Eiko Endo, Hidee Fujimoto, Akira Ishimori, Noboru \\ Matsuura, ${ }^{*}$ Juneji Khato and Tohru Takahashi* \\ Department of Clinical and Laboratory Medicine, Tohoku \\ University School of Medicine, and *Department of \\ Pathology, Research Institute for Tuberculosis and Cancer, \\ Tohoku University, Sendai 980
}

\begin{abstract}
Endo, E., Fujimoto, H., Ishimori, A., Matsuura, N., Кнатo, J. and Takahashi, T. In Vitro Test of Phagocytic Ability of Human Platelets Using Colloidal Carbon. Tohoku J. exp. Med., 1984, 142 (2), 131-139_ A simple and reproducible test for phagocytic ability of human platelets are described. Platelets obtained from heparinized venous blood were incubated with colloidal carbon (particle diameter $=0.11 \mu \mathrm{m}$ ) in autologous plasma at $37^{\circ} \mathrm{C}$. The number of platelets with or without carbon particles on smear preparations was determined at fixed intervals. Electron microscopic observation confirmed the apparent inclusion of carbon particles in platelets as being an internalization of these particles in the vesicular or canalicular structures. Under optimum conditions, the percentage of phagocytosis-positive platelets in healthy donors was $66.6 \pm 14.3$ (S.D.) $\%$ and the reproducibility of the test was considered to be satisfactory. There was no significant difference between men and women. — phagocytosis ; human platelets; colloidal carbon
\end{abstract}

Uptake of various particles by platelets is a widely recognized phenomenon. Ultrastructural studies have demonstrated uptake of Thorotrast (David-Ferreira 1964 ; White 1968; Mant and Firkin 1972; Mustard and Packham 1968), latex particles (Movat et al. 1965; White 1972), and bacteria (Clawson 1973) by platelets.

Lewis et al. (1976) have reported the isolation of polystyrene latex particles in acid phosphatase-positive vacuoles in platelets. White and Clawson (1981, 1982) have reported that channels of the open canalicular system of platelets serve as the major route for latex particle ingestion.

The number, and ability of adhesion and aggregation of platelets have been routinely tested clinically. However, the clinical significance of the phagocytic ability of platelets or their relation to pathophysiological conditions is not yet

Received for publication, May 24, 1983.

Reprint request to: Eiko Endo, Department of Clinical and Laboratory Medicine,

Tohoku University School of Medicine, 1-1 Seiryo-machi, Sendai 980. 
clear.

The purpose of the experiments described below was to develop a quantitative test of phagocytic ability of platelets using colloidal carbon.

\section{Materials and Methods}

Platelet preparation. Blood samples of healthy volunteers were taken by means of plastic syringes and immediately transferred to plastic centrifuge tubes containing heparin $(10 \mathrm{U} / \mathrm{ml})$ as an anticoagulant. The number of platelets in the platelet-rich plasma (PRP) was determined using a Coulter Counter (model S-Plus II, Coulter Electronics, Inc., Hialeah, Florida, USA) and the suspension was diluted with autologous platelet-poor plasma (PPP). To examine the influence of plasma concentration on phagocytosis, the suspension was further diluted with Hanks' balanced salt solution containing $\mathrm{Ca}^{++}$and $\mathrm{Mg}^{++}$(HBSS).

Incubation of platelets with colloidal carbon. A suspension of colloidal carbon (Pelican drawing ink, Hanover, Germany) was diluted with PPP and prewarmed at $37^{\circ} \mathrm{C}$ in a plastic tube ( $12 \mathrm{~mm}$ in diameter). To $0.05 \mathrm{ml}$ of this suspension, $0.45 \mathrm{ml}$ of the platelet suspension was added and gently agitated in the tube, which was then incubated at $37^{\circ} \mathrm{C}$ without stirring. The suspension was gently stirred before sampling and $0.005 \mathrm{ml}$ samples were taken at fixed intervals. The samples were spread on glass slides and stained with MayGiemsa solution.

Electron microscopic examination. The procedure followed that of Clawson (1973) and White and Clawson (1982). Briefly, the samples were fixed in suspension by adding an

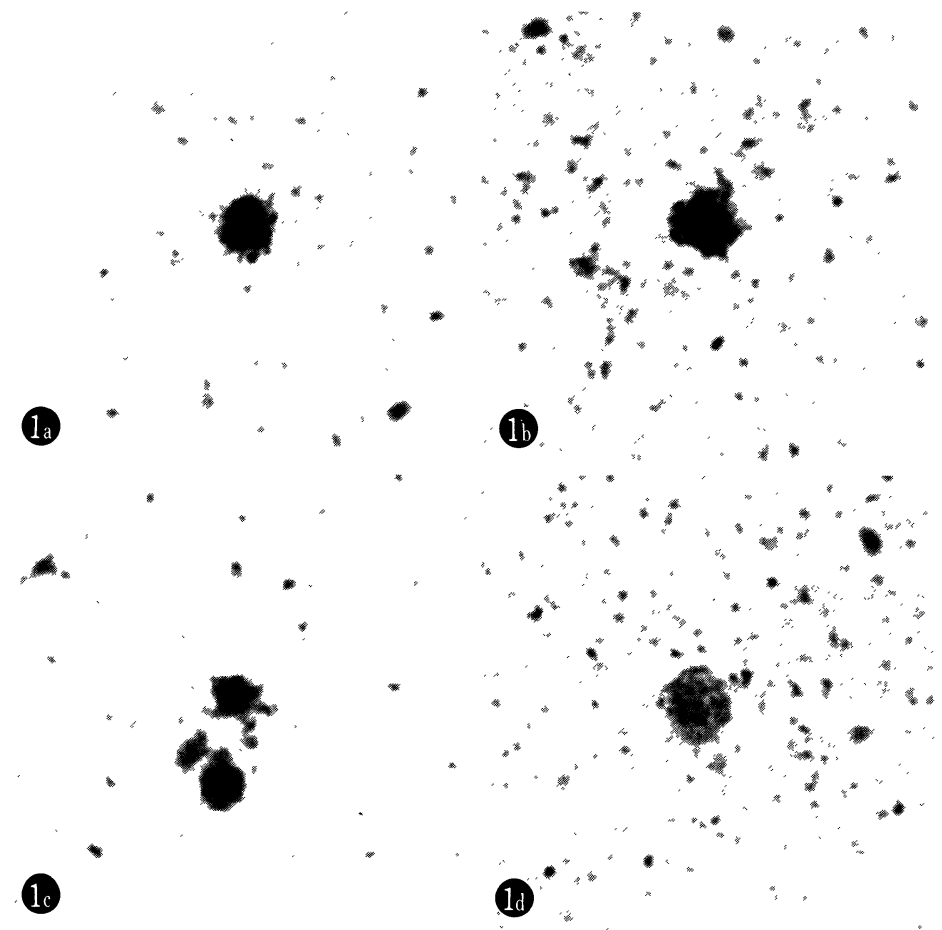

Fig. 1. Light microscopic appearance of platelets. Incubation for $30 \mathrm{~min}$. Giemsa stain. $\times 2,000$. The carbon particles contained in the platelet were aggregated and had a linear pattern ( $a$ and $b)$. The phagocytosis-positive cells tend to aggregate with other platelets (c). A phagocytosis-negative platelet is shown in $d$. 
equal volume of $0.2 \%$ glutaraldehyde and $0.16 \%$ paraformaldehyde in $0.1 \mathrm{M}$ cacodylate$\mathrm{HCl}$ buffer $\left(\mathrm{pH} \mathrm{7.4). \quad After} 15 \mathrm{~min}\right.$ at $37^{\circ} \mathrm{C}$, the samples were centrifuged at $810 \times \mathrm{g}$ for 15 min at room temperature and the sediment was fixed in $2.5 \%$ glutaraldehyde and $2 \%$ paraformaldehyde in a buffer. The resulting button of cells was fixed again in 1\% osmium tetroxide in buffer for $2 \mathrm{hr}$ at $4^{\circ} \mathrm{C}$. Following dehydration in graded ethyl alcohol, the fragments of the button were embedded in Epok 812 (Ohken Shoji, Ltd., Tokyo). Sections, $1 \mu \mathrm{m}$ thick, were cut and stained with toluidine blue. After selecting an appropriate area with the light microscope, ultrathin sections, $65 \sim 75 \mathrm{~nm}$ in thickness, were cut and placed on a copper grid precoated with $0.25 \%$ Bioden Meshcement (Neoprene solution in toluene, Ohken). Then these sections were doubly contrasted with uranium acetate and lead nitrate and examined by means of an electron microscope (Hitachi, Model H 300).

Determination of phagocytic ability of platelets. The platelets which contained a concentration of carbon particles were designated as phagocytosis-positive (Fig. 1a, b, c). The plațelets in which carbon particles were spread sparsely but not concentrated were designated as negative (Fig. 1d). The total number of platelets counted on a smear preparation was 200 and the percentage of positive cells was calculated. In cases where judgement was difficult, the decision was based on the consensus of the three examiners.

\section{Results}

The preliminary experiments employing a concentration of platelets higher than $10^{5} / \mathrm{mm}^{3}$ resulted in an aggregation of platelets during incubation. When sodium citrate was used instead of heparin, the aggregation was somewhat impeded; however, it was still difficult to distinguish the individual cells on the smear preparation.

In the first series of experiments, the effect of the concentration of colloidal

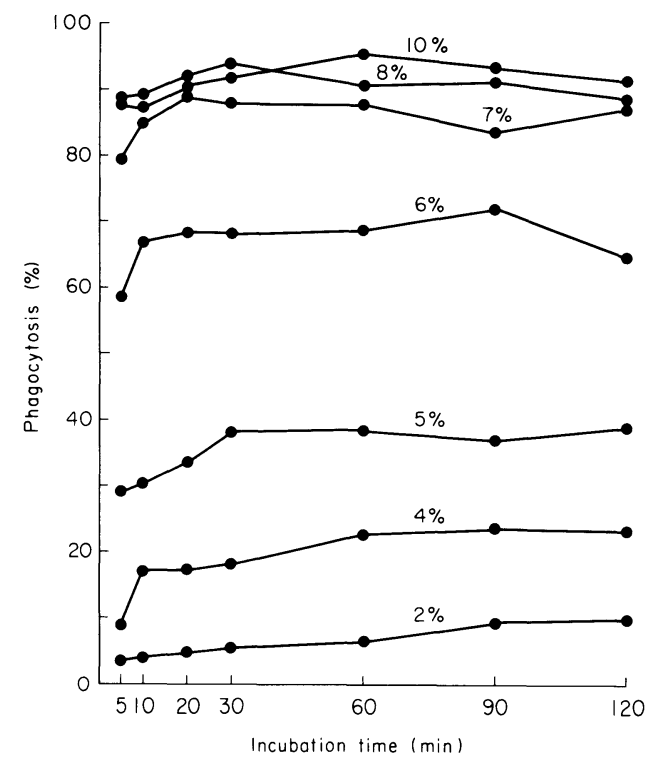

Fig. 2. Influence of the concentration of colloidal carbon and incubation time on phagocytic ability of platelets. The suspension of platelets $\left(5 \times 10^{4} / \mathrm{mm}^{3}\right)$ was incubated with that of colloidal carbon at $37^{\circ} \mathrm{C}$. Smear preparations were made at various incubation times and the number of platelets with or without carbon particles was determined. 
carbon and time of incubation on phagocytosis were studied (Fig. 2).

The percentage and degree of phagocytosis increased proportionally with an increase in colloidal carbon concentration. With a concentrations of $8-10 \%$ colloidal carbon, almost $90 \%$ of platelets included particles within 5 min, while with $2-4 \%$ colloidal carbon, the percentage of platelets with carbon particles remained very low. After incubation for $30 \mathrm{~min}$, the percentage of phagocytosis did not change markedly any more; therefore an incubation time of 30 min was selected in the following experiments.

Observations with a light microscope suggested that the concentration of particles to the platelet was not due to attachment to the cell surface but to phagocytosis, because some of the particles were seen as aggregates and some formed thin linear structures (Fig. la, b). The black particles were easily dis-

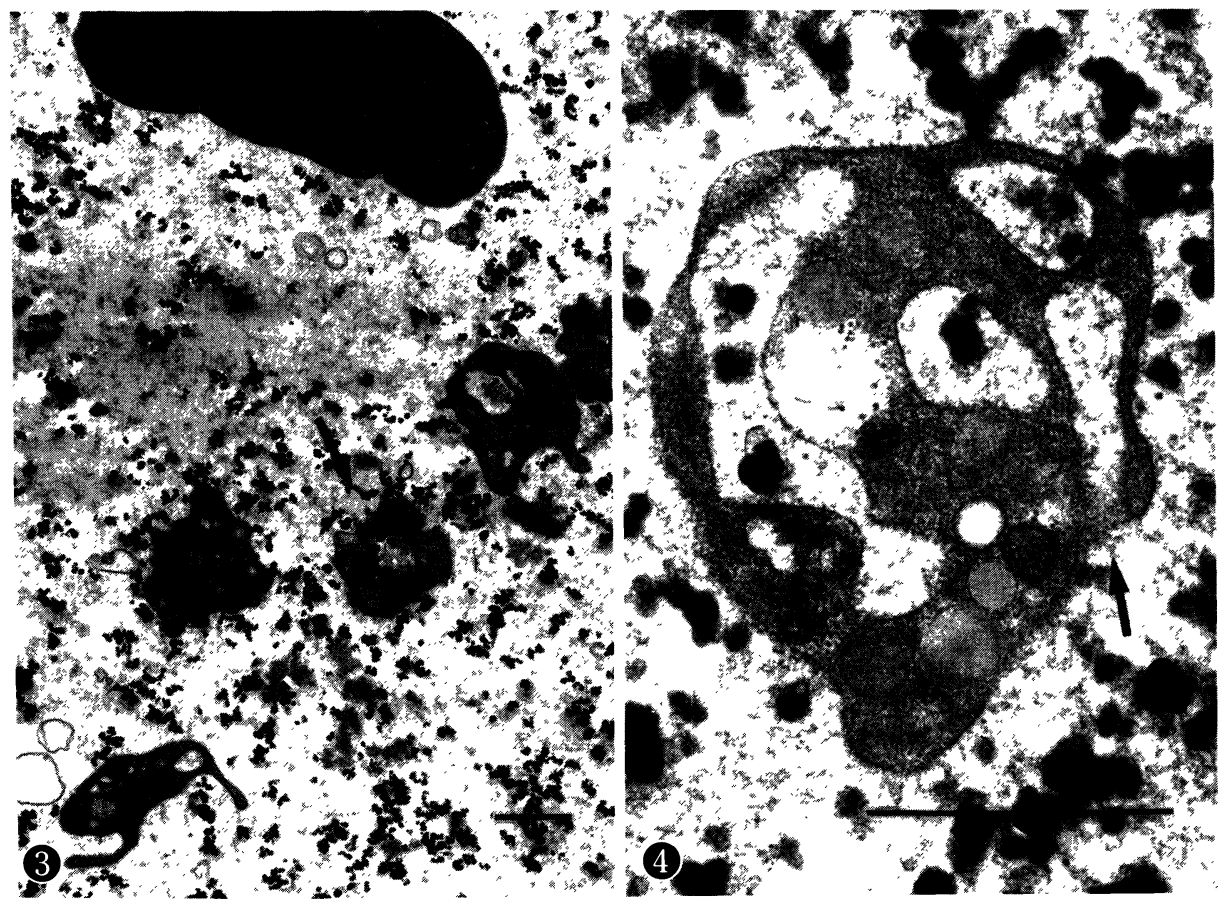

Fig. 3. Electron microscopic appearance of platelets. Incubation for $30 \mathrm{~min} . \quad \times 7,800$. The phagocytosis-positive cell (arrow) contained a number of carbon particles in the vesicle. In all electron micrographs calibration bars indicate $1 \mu \mathrm{m}$.

Fig. 4. Electron microscopic appearance of platelets. Incubation for $30 \mathrm{~min} . \times 31,500$. Carbon particles are seen in the canalicular structures which seem to open to the cell surface (arrow).

Fig. 5. Electron microscopic appearance of platelets. Incubation for $1 \mathrm{hr}$. $\times 15,800$. The vesicles containing carbon particles are markedly enlarged.

Fig. 6. Electron microscopic appearance of platelets. Incubation for $1 \mathrm{hr}$. $\times 23,600$. Some phagocytosis-positive platelets show degenerative ehanges such as vague configuration and elongation or irregular morphology of platelets. 


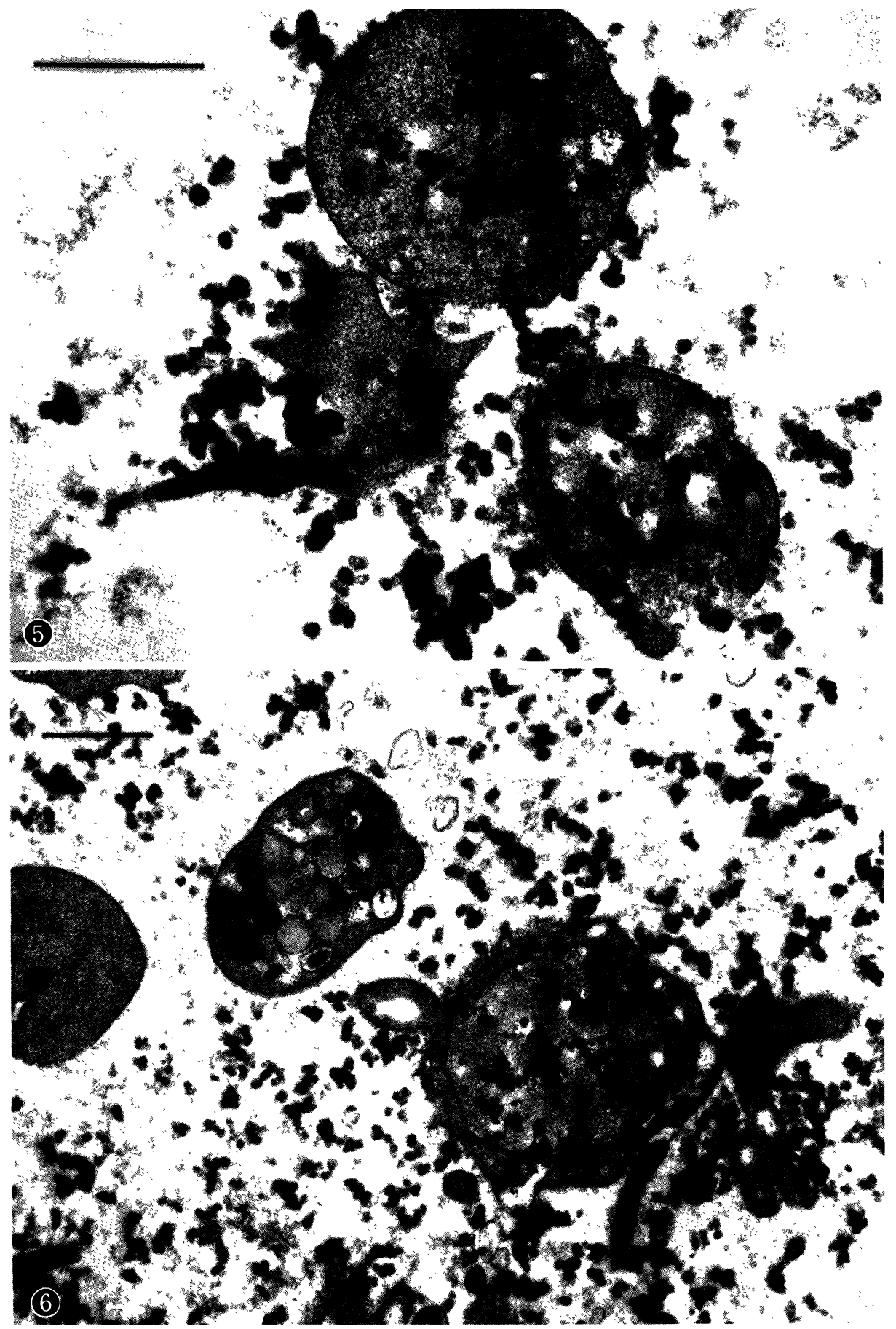


tinguished from the azurophilic granules.

Some of the platelets which included particles aggregated with other platelets (Fig. 1c). The degeneration of cells, suggested by stretching or elongation and vague configurations of cells, was observed after incubation longer than $60 \mathrm{~min}$.

Electron microscopic examination revealed that the carbon particles were contained within the vesicles. As seen in Fig. 3, the carbon particles, $0.11 \pm 0.02$ $\mu \mathrm{m}$ in diameter, were isolated in intracellular vesicles. Specific attachment of carbon particles to the plasma membrane was not noted. In some platelets the particles were localized in the canalicular space (Fig. 4). The vesicles containing the particles seemed to enlarge with time (Figs. 5 and 6), while the enlargement of vesicles in the phagocytosis-negative platelets was less remarkable.

In the next series of experiments, the influence of plasma concentration on phagocytosis was studied. Table 1 summarizes the results. Two samples of platelets prepared from different donors were used. The percentage and degree of phagocytosis seemed to increase when platelets were incubated in lower concentrations of plasma. When incubated in a medium with a plasma concentration lower than $50 \%$, it was difficult to distinguish the individual platelets because of the aggregation and degeneration. Table 2 represents the normal values of phagocytic ability of platelets for men and women determined using a $6 \%$ concentration of colloidal carbon with incubation for $30 \mathrm{~min}$. The age of donors

TABLE 1. Influence of plasma concentration on phagocytic ability of platelets

\begin{tabular}{|c|c|c|c|c|c|c|c|c|}
\hline & & \multicolumn{7}{|c|}{ Plasma concentration $(\%)$} \\
\hline & & \multicolumn{2}{|c|}{100} & \multicolumn{2}{|c|}{75} & \multicolumn{2}{|c|}{50} & 25 \\
\hline & & Case 1 & Case 2 & Case 1 & Case 2 & Case 1 & Case 2 & Case 1 Case 2 \\
\hline \multirow[t]{2}{*}{$\begin{array}{l}\text { Phagocytosis } \\
(\%)\end{array}$} & $\begin{array}{l}30 \text { min } \\
\text { incubation }\end{array}$ & 34.3 & 30.1 & 47.0 & 58.8 & 92.8 & 94.5 & N.D. N.D. \\
\hline & $\begin{array}{l}60 \text { min } \\
\text { incubation }\end{array}$ & 38.8 & 29.3 & 54.3 & 60.8 & N.D. & N.D. & N.D. N.D. \\
\hline
\end{tabular}

The plasma was diluted with Hanks' balanced salt solution.

N.D., not determined because of aggregation and degeneration of platelets.

TABLE 2. Normal value of phagocytic ability of platelets for men and women

\begin{tabular}{lcc}
\hline & $n$ & Mean \pm s.D. $(\%)$ \\
\hline Men & 11 & $65.1 \pm 11.5$ \\
Women & 10 & $68.1 \pm 17.5$ \\
Total & 21 & $66.6 \pm 14.3$ \\
\hline
\end{tabular}

Concentration of platelets and colloidal carbon were $5 \times 10^{4} / \mathrm{mm}^{3}$ and $6 \%$, respectively. The mixture was incubated at $37^{\circ} \mathrm{C}$ for $30 \mathrm{~min}$. 
TABLE 3. Reproducibility test of phagocytic ability of platelets

\begin{tabular}{cc}
\hline Test number & Phagocytosis $(\%)$ \\
\hline 1 & 71.0 \\
2 & 68.5 \\
3 & 63.5 \\
4 & 63.5 \\
5 & 61.5 \\
6 & 66.3 \\
7 & 66.5 \\
8 & 65.5 \\
9 & 61.0 \\
10 & 64.0 \\
Mean \pm S.D. & $65.1 \pm 3.1$ \\
s.D. & 3.1 \\
C.V. & 4.1 \\
\hline
\end{tabular}

Phagocytic ability of platelets obtained from the same donor was determined repeatedly under the same conditions as utelized in Table 2. C.V., coefficient of variation.

ranged from 25 to 45 years. The mean values for men and women were 65.1 and $68.1 \%$ respectively, and there was no significant difference between them. There was a relatively wide distribution of normal values (S.D. $=14.3 \%$ ), especially in women $($ s.D. $=17.5 \%)$. Table 3 indicates the reproducibility of the test. It appears that the variation of values obtained by repeated tests for the same donor was very small $(3.1 \%)$.

\section{Discussion}

The number, and ability of adherence and aggregation of platelets are considered to be important physiological functions of platelets related to hemostasis. Although it is well known that platelets take up a wide variety of foreign substances in vivo and in vitro, little work has been done about the quantitative significance of phagocytosis under pathophysiological conditions.

Chang and Hamilton (1979) described a direct counting method for the determination of phagocytic ability of chicken platelets using the bacteria, Enterobacter cloacae. They reported that platelets phagocytized 3 times as rapidly as heterocytes or monocytes and suggested the primary role of platelets in the clearance of foreign particulates.

The phagocytosis of colloidal carbon by chicken platelets has been reported by Awadhira et al. (1980). They injected a suspension of colloidal carbon intravenously and observed a tendency of platelets containing the particles to aggregate, which was followed by a progressive reduction in the number of 
platelets in the circulating blood.

The association of phagocytosis and aggregation of platelets has been reported by Movat et al. (1965), who added latex particles to platelet suspensions. They noted that adenosine and adenosine monophosphate, which were known to inhibit platelet aggregation induced by adenosine diphosphate, blocked the aggregation but did not prevent phagocytosis of latex particles by platelets.

The authors examined conditions affecting platelet aggregation and found that a dilution of platelet suspension successfully reduced the aggregation without reducing phagocytosis. It also appeared that agitation of the test tube during incubation enhanced the aggregation.

The authors tried several kinds of colloidal carbon including special india ink (C11/1431a, Gunther Wagner Perikan Werke, Hanover, Germany) which has been used by previous authors for studies on phagocytosis of platelets (Movat et al. 1965 ; Tennent and Donald 1976; Awadhiya et al. 1980). It was found that drawing ink was the most satisfactory for quantitative testing purposes because the particles in it are relatively uniform in size.

White (1968) reported that the incubation of platelets with thorotrast particles at $37^{\circ} \mathrm{C}$ resulted in an internalization of the particles in the canalicular system within $5 \mathrm{~min}$ without any alteration in platelet morphology. However, incubation longer than $15 \mathrm{~min}$ caused dilatation of thorotrast-filled canaliculi, distortion of external contour, and discharge of some granules. Lewis et al. (1976) have suggested that platelets may form sealed phagocytic vesicles in which latex particles and acid phosphatase are localized. We noted an enlargement of vesicles in phagocytosis-positive cells, which was more marked than in phagocytosis-negative platelets.

Mant et al. (1972) compared uptake of latex and thorotrast particles by human platelets in vitro. They reported that the uptake of latex particles was probably by an active phagocytic process, which required energy from anerobic glycolysis and the presence of divalent cations, while thorotrast uptake was by a different mechanism, since energy requirements for thorotrast uptake were minimal and divalent cations were not needed. They suggested that other very small particles, such as ferritin and viruses, might be taken up by the same process as thorotrast.

The mechanism of the increase in phagocytic ability noted when platelets were incubated in lower concentrations of plasma is unknown. It has been reported that lack of plasma protein in the platelet suspension leads to increased adherence of the platelets to the glass surface and precoating of the glass surface with plasma proteins such as fibrinogen, albumin and gammaglobulin affects the adherence, aggregation and release of platelet contents (Packham et al. 1969). Further work is needed to clarify the factors controlling phagocytosis of platelets and also to clarify the interaction with other types of phagocytic cells such as neutrophils, monocytes and macrophages in the process of clearance of foreign 
particulates from circulating blood.

\section{References}

1) Awadhiya, R.P., Vegad, J.L. \& Kolte, G.N. (1980) Demonstration of the phagocytic activity of chicken thrombocytes using colloidal carbon. Res. Vet. Sci., 29, 120-122.

2) Chang, F.-F. \& Hamilton, P.B. (1979) The thrombocytes as the primary circulating phagocyte in chickens. J. reticuloend. Soc., 25, 585-590.

3) Clawson, C.C. (1973) Platelet interaction with bactiria III. Ultrastructure. Amer. J. Path., 70, 449-472.

4) David-Ferreira J.F. (1964) The blood platelet: electron microscopic studies. Int. Rev. Cytol., 17, 99-148.

5) Lewis, J.C., Maldonado, J.E. \& Mann, K.G. (1976) Phagocytosis in human platelets : Localization of acid phosphatase-positive phagosomes following latex uptake. Blood, 47, 833-840.

6) Mant, M.J. \& Firkin, B.G. (1972) Uptake of latex and thorotrast by human platelets in vitro : effect of various chemicals demonstrating differing mechanisms and metabolic requirements. Brit. J. Haematol., 22, 383-391.

7) Movat, H.Z., Weiser, W.J., Glynn, M.F. \& Mustard, J.F. (1965) Platelet phagocytosis and aggregation. J. Cell Biol., 27, 531-543.

8) Mustard, J.F. \& Packham, M.A. (1968) Platelet phagocytosis. Series Haematol., 1, 168-184.

9) Packham, M.A., Evans, G., Glynn, M.F. \& Mustard, J.F. (1969) The effect of plasma proteins on the interaction of platelets with the glass surface. J. Lab. clin. Med., 73, 686-697.

10) Tennent, R.J. \& Donald, K.J. (1976) The ultrastructure of platelets and macrophages in particle clearance stimulated by zymosan. $J$. reticuloend. Soc., 19, 269-280.

11) White, J.G. (1968) The transfer of thorium particles from plasma to platelets and platelets granules. Amer. J. Path., 53, 567-575.

12) White, J.G. (1972) Uptake of latex particles by blood platelets; phagocytosis or sequestration. Amer. J. Path., 69, 439-458.

13) White, J.G. \& Clawson, C.C. (1981) Effects of large latex particle uptake on the surface connected canalicular system of blood platelets: A freeze-fracture and cytochemical study. Ultrastruct. Path., 2, 277-287.

14) White, J.G. \& Clawson, C.C. (1982) Effects of small latex particle uptake on the surface connected canalicular system of blood platelets: A freeze-fracture and cytochemical study. Diag. Histopath., 5, 3-10. 